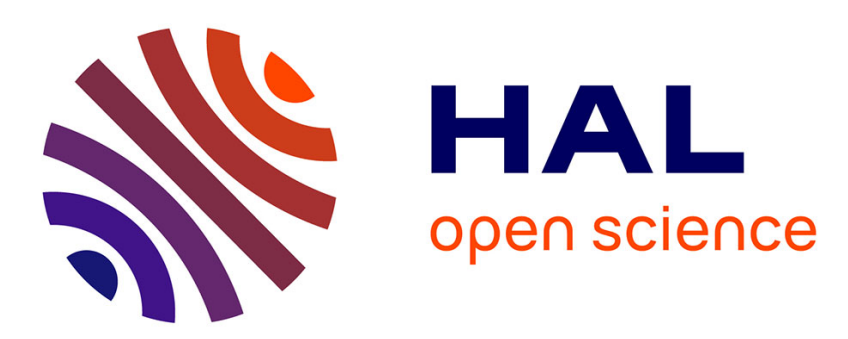

\title{
Factions et spectacles de l'hippodrome dans les papyrus grecs à Hermou polis de Thébaïde. Etude préliminaire
}

Michel Matter

\section{To cite this version:}

Michel Matter. Factions et spectacles de l'hippodrome dans les papyrus grecs à Hermou polis de Thébaïde. Etude préliminaire. KTĖMA Civilisations de l'Orient, de la Grèce et de Rome antiques, 1996, 21, pp.151-156. halshs-00003892v2

\section{HAL Id: halshs-00003892 \\ https://shs.hal.science/halshs-00003892v2}

Submitted on 18 May 2005

HAL is a multi-disciplinary open access archive for the deposit and dissemination of scientific research documents, whether they are published or not. The documents may come from teaching and research institutions in France or abroad, or from public or private research centers.
L'archive ouverte pluridisciplinaire HAL, est destinée au dépôt et à la diffusion de documents scientifiques de niveau recherche, publiés ou non, émanant des établissements d'enseignement et de recherche français ou étrangers, des laboratoires publics ou privés. 


\section{Factions et spectacles de l'hippodrome dans les papyrus grecs à Hermou polis de Thébaïde Étude préliminaire}

Résume. - Présentation préliminaire d'un papyrus grec inédit (première moitié du $\mathrm{VI}^{\mathrm{e}} \mathrm{s}$.) provenant Hermopolis Magna, conservé à la Bibliothèque Nationale de Vienne (Autriche), appartenant à un codex et comportant, parmi une liste de dépenses publiques, celle qui est relative aux chevaux de l'hippodrome ; c'est la première mention papyrologique de ce monument destiné aux spectacles, connu par ailleurs par des indications indirectes (factions) qui ne l'avaient jamais encore nommé expressis uerbis.

ZuSAMmenfassung. - Vorbemerkungen über einen unveröffentlichen, aus einem Kodex stammenden grieschischen Papyrus (erste Hälfte des vı. Jh.) aus Hermopolis Magna, mit dem ersten papyrologischen Beleg des städtischen Hippodroms, bezüglich Ausgaben, bei Erwährung derjenigen für die Rennpferde.

À la différence des autres hippodromes connus en Égypte romaine et byzantine ( ${ }^{1}$ ), celui d'Hermopolis $\left({ }^{2}\right)$ est attesté de manière indirecte (mentions des factions et de courses) par un nombre relativement conséquent de papyrus, une dizaine dans l'état actuel de la documentation, mais par aucun vestige archéologique. Son emplacement exact n'est pas même connu. L'édifice proprement dit, jamais mentionné par les papyrus, l'est par une inscription ( ${ }^{3}$ ) ; son existence est cependant fondée par des indications sur les spectacles (courses de chevaux) qui s'y sont

(1) J. H. Humphrey, Roman Circuses. Arenas for Chariot Racing, Londres, 1986, pp. 505-520 ; D. M. Balley, "Classical Architecture in Roman Egypt", in M. Hevig éd., Architecture and Architectural Sculpture in the Roman Empire, Oxford, 1990, p. 123 ; The Oxford Dictionnary of Byzantium, 2, 1991, pp. 934-936, s.u. "Hippodromes"; un hippodrome est connu depuis peu sur le site de Péluse (cf. Cahiers de Recherches de l'Institut de Papyrologie et d'Égyptologie de Lille, 16, 1994, p. 117).

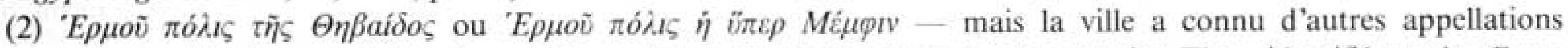
au cours de son histoire - est l'ancienne Hmnw d'époque pharaonique, la ville du dieu Thot, identifié par les Grecs à Hermès. Hermou polis Magna (nous adoptons, par commodité, la graphie Hermopolis) est la métropole du nome hermopolite, en Moyenne Égypte, qui occupait les deux rives du Nil sur environ $120 \mathrm{~km}$ de longueur, depuis le sud, à la frontière du nome lycopolite, jusqu'au nord, au voisinage des nomes cynopolite et oxyrhynchite. (M. DREWBEAR, Le nome hermopolite. Toponymes et sites, American Studies in Papyrology, 21, Ann Arbor, 1979, pp. 45-49). Pour les différentes appellations du chef-lieu du nome, voir N. Litinas, «Hermou polis of the Thebais. Some Corrections and Notes Concerning its Name and Epithets", Archiv für Papyrusforschung, 41, 1995, pp. 66-84. Le nom grec de

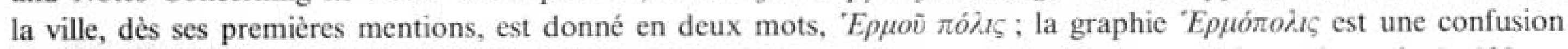

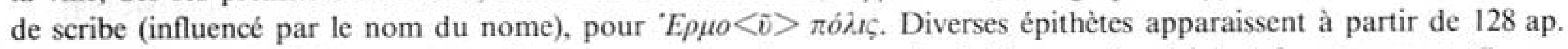
J.-C. La ville était divisée, semble-t-il, en quatre quartiers au moins à l'époque impériale (cf. notamment Corpus Papyrorum Hermopolitanorum 127 = P. Vindob. Gr. 12565 (Gallien), car des papyrus byzantins (par ex. BGU XII 2201 (565) indiquent d'autres divisions de la cité). Les papyrus d’époque arabe connaissent toujours cette division majeure en quatre quartiers, mais ne nous apprennent par ailleurs rien sur l'hippodrome.

(3) E. BERNAND, Inscriptions métriques de l'Égypte gréco-romaine. Recherches sur la poésie épigrammatique des Grecs en Égypte, Paris, 1969, $\mathrm{n}^{\circ} 97=$ SEG, VIII, $621=S B, \mathrm{IV}, 7871$. 
déroulés et par des données d'ordre topographique. En outre, dès la haute époque byzantine ( $\left.{ }^{4}\right)$, apparaissent dans les papyrus des mentions aux factions des Bleus et des Verts de l'hippodrome.

Bien que le papyrus - inédit - dont il va être question $\left(^{5}\right)$ ne mentionne ni faction ni dème, mais des chevaux pour les courses, il n'est pas inutile de rappeler brièvement ce que recouvrent ces notions, en rapport avec les spectacles de l'hippodrome. La question des factions, souvent controversée, a fait l'objet de discussions $\left(^{6}\right)$ auxquelles A. Cameron $\left(^{7}\right)$ a donné un tournant décisif.

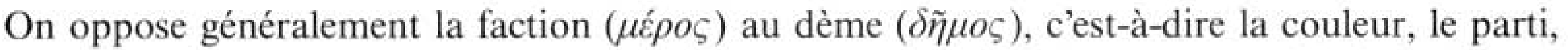
à l'unité topographique (quartier) d'une ville $\left(^{8}\right)$; cette distinction est toutefois inégalement acceptée - notamment par A. Cameron qui ne s'est appuyé que sur des sources littéraires et principalement sur l'exemple de Constantinople. Cependant, «les sources papyrologiques, documentation éminemment concrète et pratique» $\left({ }^{9}\right)$, ne montrent, dans leur état actuel, jamais

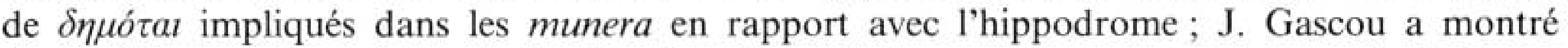

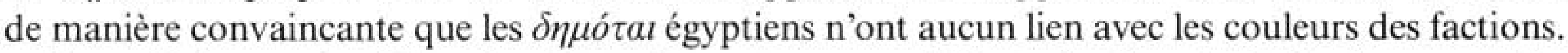

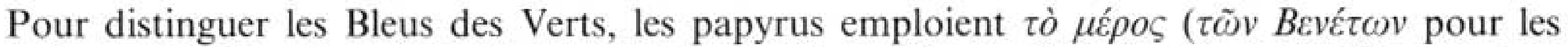

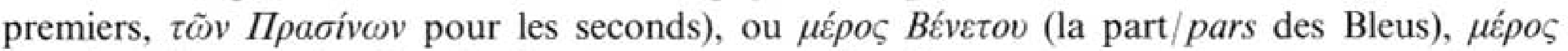

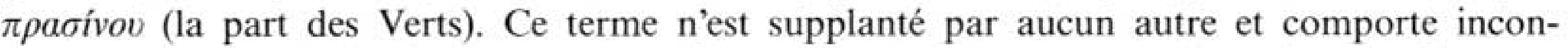
testablement une indication de topographie urbaine, dans le cas des métropoles possédant un hippodrome.

De manière générale, l'hippodrome est connu très précocement dans un certain nombre de villes égyptiennes. À Oxyrhynchus, dès 22-25 (P. Oxy. II 288); ailleurs, les papyrus en attestent dès la fin de l'époque ptolémaïque, notamment à Hérakléopolis et à Memphis où sont mentionnés des naukleroi hippodromitai en 64/63 avant J.-C. (BGU VIII 1741-1743). Antinoopolis possédait un hippodrome monumental $\left({ }^{10}\right)$ et à Hermopolis des courses de chevaux sont attestées en 195 (P. Ryl. II 86). Alexandrea ad Aegyptum, que nous mettrons à part, a peut-être possédé deux hippodromes, à en juger d'après un épineux problème de vocabulaire pour désigner ces monuments (11). Mais l'hippodrome a connu une grande faveur surtout aux $\mathrm{IV}^{\mathrm{e}}-\mathrm{VI}^{\mathrm{e}}$ siècles, liée certainement à la quasi inexistence - sauf à Alexandrie - d'autres formes de spectacles comme les munera ou les uenationes de l'amphithéâtre $\left({ }^{12}\right)$; cette faveur de l'hippodrome peut être due aussi à la faible diffusion de représentations proprement théâtrales $\left({ }^{13}\right)$.

(4) À partir du $\mathrm{IV}^{e} \mathrm{~S}$., puis, avec une nette prédominance, aux $\mathrm{vi}^{\mathrm{e}}$ et $\mathrm{vII}^{\mathrm{e}} \mathrm{S}$. Pour des détails sur les aspects techniques de l'hippodrome et le fonctionnement, p.ex., de celui d'Oxyrhynchus, voir O. Ashm. Shelton 83-190 (Iv's.).

(5) Jean Gascou, avec la permission de Hermann Harrauer, directeur de la Papyrussammlung der österreichischen Nationalbibliothek (Vienne), m'a confié la publication de ce lot de papyrus, qui fait partie d'un codex fiscal : P. Vindob. Gr. $15253+14323+31036+40970+23866$

(6) Depuis l'étude de G. Manajlovic, "Le peuple de Constantinople», Byzantion, 11, 1936, pp. $617-716$ (ed. pr. en serbo-croate en 1904) qui a pour ainsi dire identifié les couleurs des factions du cirque à des partis politiques.

(7) A. Cameron, Porphyrius the Charioteer, Oxford, 1973 et Circus Factions. Blues and Greens at Rome and Byzantium, Oxford, 1976.

(8) J. Gascou, «Les institutions de l'hippodrome en Égypte byzantine», BIFAO, 76, 1976, pp. 185-212 (186-189).

(9) Ibid., p. 186.

(10) Description de l'Égypte, Antiquités, IV, 1822, p. 16 sq., pl. 437 et 438 ; D. M. BAlLEy, loc. cit., p. 123.

(11) J. H. Humphrey, op. cit., pp. 505-512; F. Burkhalter, «Le gymnase d'Alexandrie : centre administratif de la province romaine d'Égypte», $B C H, C X V I, 1992$, pp. 345-373.

(12) Les papyrus ne mentionnent jamais l'amphithéâtre. STRABos, 17, 1, 10 en connait un à Nicopolis, à l'est d'Alexandrie ; celui que Charles-Jacques Poncet aurait vu pendant son voyage en Égypte en 1698 à Lycopolis (cf. S. SAuneron, "L'amphithéâtre d'Assiout", Villes et légendes d'Égypte, IFAO, Le Caire, 19832, pp. 90-94) est très 
Le fragment de codex P. Vindob. G 15253, inédit, est un papyrus opisthographe conservé à la Bibliothèque Nationale de Vienne (Autriche) et fait partie des importants lots de papyrus achetés à la fin du $\mathrm{XIX}^{\mathrm{e}}$ et au début du $\mathrm{xx}^{\mathrm{e}} \mathrm{s}$. en Égypte $\left({ }^{14}\right)$. Seule la ligne 12, fragmentaire, du recto a trait à l'hippodrome. Après une brève description commentée du papyrus et du contenu de cette ligne, on établira, en second lieu, un dossier préliminaire des attestations hermopolitaines - et l'unique mention épigraphique - relatives à l'hippodrome et aux couleurs des factions.

Le papyrus ne comporte pas de datation précise, mais le style, le formulaire et des critères paléographiques permettent de l'attribuer à la première moitié du $\mathrm{vi}^{\mathrm{e}} \mathrm{s}$. Il s'agit d'une liste

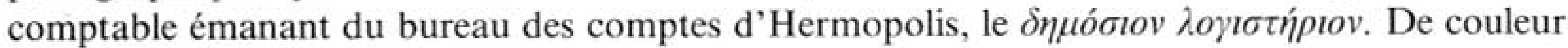
brune, le papyrus $(15 \times 22 \mathrm{~cm})$ est amputé sur tous les côtés, principalement dans sa partie inférieure (une $\kappa o \lambda \lambda \dot{\eta} \sigma l \varsigma$ est visible à $2 \mathrm{~cm}$ environ du bord droit). Cette liste comptable donne, à gauche, les entrées, c'est-à-dire qu'elle énumère, sous forme de liste, des rubriques pour lesquelles des sommes sont à engager. Ces montants chiffrés, quand ils sont conservés, figurent à droite ; certaines sommes, biffées, sont corrigées et le scribe a grand soin d'être le plus précis possible. Il note - et c'est une curiosité - tantôt un nomismation, tantôt 24 carats, ce qui, à quelques variantes près, est sensiblement équivalent ; en tout cas, l'usage du carat donne, d'après la documentation papyrologique actuelle, l'année 433 comme terminus post quem (P. Amst. I 53). Selon une pratique mal comprise mais courante à l'époque tardive, il note aussi, à plusieurs reprises, une somme chiffrée en nomismata minorés d'un certain nombre de keratia (p.ex. 18 nom. moins 4 keratia, au lieu d'écrire 17 nom. 20 keratia). Sans entrer dans des questions de fiscalité byzantine en Égypte, notons que l'emploi du sou d'or minoré de tant et tant de carats est un difficile problème de l'économie antique (15), particulièrement dans les papyrus d'Égypte.

À deux reprises et dès les premières lignes, on indique que les sommes requises pour le

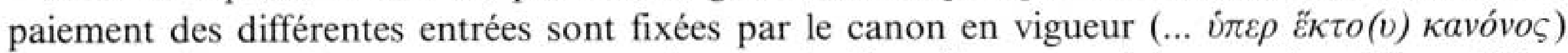
et cette référence à un canon, $i . e$. un impôt régulier, fixe, est précieuse puisqu'elle entérine un état de fait et sert de modèle législatif. Les entrées sont très diverses: on énumère de la nourriture destinée aux chameaux, des travaux à effectuer, toujours selon le canon, sur une digue, ou encore de l'huile pour l'éclairage public $\left({ }^{16}\right)$.

hypothétique ; il en est de même d'Arsinoé-Crocodilopolis du Fayoum (V. Poulsen, Les portraits romains, 1, Copenhague, 1962, nos 32, 34 et 45 à propos de portraits d'Auguste, Livie et Tibère provenant prétendûment de l'amphithéâtre (?) d'Arsinoé, où il semble plutôt s'agir d'une exèdre).

(13) Nous avons entrepris un relevé systématique, essentiellement papyrologique, mais aussi archéologique et littéraire, des édifices destinés aux spectacles et des spectacles proprement dits (jeux, concours, diverses manifestations agonistiques, etc.) en Égypte romaine. Pour le théâtre, citons seulement, à titre d'exemple, Oxyrhynchus où un papyrus (P. Oxy. XVII 2128) indique un montant de 3500 drachmes pour l'achat (?) du velum du théâtre (fin $\Perp^{e} \mathrm{~s}$.) dans un compte de dépenses municipales.

(14) L'histoire de ces achats de papyrus, par des universités et des bibliothèques d'Europe et d'Amérique, est retracée dans K. Preisendanz, Papyrusfunde und Papyrusforschung, Leipzig, 1933, ainsi que dans P. Rainer Cent. (1983), pp. 3-93.

(15) M. F. Hendy, Studies in the Byzantine Monetary Economy c. 300-1450, Cambridge, 1985; ainsi que l'a montré en particulier K. MARESCH, Nomisma und Nomismatia. Beiträge zur Geldgeschichte Ägyptens im 6. Jahrhundert n. Chr., Opladen, 1994, p. 5 sq.

(16) À ce propos, il convient de relever brièvement (la question sera approfondie lors de la publication du codex), que ce type d'ćclairage n'est connu, dans toute la littérature antique, que pour une seule ville, Antioche-sur-l'Oronte, à propos de laquelle Ammien Marcellin, 14, 1, 9, fustigeant le comportement du César Gallus (351-354), la nuit dans les rues de la ville, écrit : et haec (Gallus questionnant les gens dans les rues d'Antioche de ce qu'ils pensent 
La ligne 12 du verso, amputée au début et à la fin, a conservé trois mots : ...]ï $\pi \pi \omega v \tau o(\widetilde{v})$ іллıкой[..., "... des chevaux de l'hippodrome...". On voit très nettement une surcharge sur $i \pi \pi \omega v$, le scribe ayant d'abord écrit ï $\pi \pi o v$, puis il a corrigé. À partir de cette ligne 12, les montants chiffrés, à droite des entrées, ne sont plus conservés; il aurait été particulièrement intéressant de connaitre les montants des sommes nécessaires à l'entretien des chevaux pour le cirque - car c'est de cela qu'il s'agit. À titre indicatif, sous Justinien (Édit XIII), un cheval vaut 750 deniers (un esclave en vaut 700); dans P. Euphr. 10 un cheval est vendu 750 deniers. Les chevaux et les courses de chars représentent une dépense importante et l'organisation de

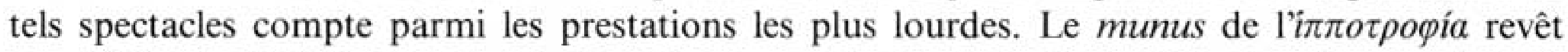
en vérité un double aspect: permanent en ce qui concerne l'entretien des chevaux (achat, nourriture, transport), et exceptionnel lors de la présentation souvent grandiose du spectacle, en vue duquel il a fallu aussi entretenir les chars et recruter les cochers. Les frais sont donc multiples.

Puisque ce texte émane du bureau des comptes publics d'Hermopolis, il est évident que l'hippodrome et ses spectacles sont financés par des revenus publics, comme le montrent d'ailleurs d'autres papyrus pour le $\mathrm{vI}^{\mathrm{e}} \mathrm{s}$., alors que des liturgies hippiques étaient encore attestées au $\mathrm{IV}^{\mathrm{e}} \mathrm{s}$. Le passage d'un système de liturgies (l'hippotrophie) à la fiscalisation du cirque, a très vraisemblablement eu lieu, sans doute par étapes, au cours du ve s., peut-être sous Anastase (491-518) (17).

Chaque fois que l'on est en présence de fondation, de transformation, d'entretien ou de restauration d'un édifice public, se pose le problème du financement de ces travaux. Le fonctionnement de ces édifices exigeait certainement des frais constants, mais il n'est pas aisé de répondre de manière précise à la question de savoir comment, dans une ville égyptienne $\left.{ }^{18}\right)$, moins homogène et probablement moins fortunée qu'une ville dans d'autres provinces orientales de l'Empire, étaient résolues les questions financières liées à la gestion des édifices publics. On sait que pour Alexandrie (Édit XIII, 15-16), en 539, Justinien demande au duc et augustal de prévoir une somme de 320 solidi pour le prix de 36 chevaux à livrer à l'hippodrome. Mais déjà en 315, le préfet d'Égypte avait requis le bourg de Karanis (Fayoum), pour fournir

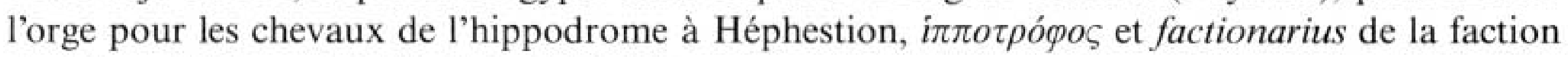
des Bleus d'Alexandrie (P. Cairo Isid. 57).

du prince) confidenter agebat in urbe, ubi pernoctantium luminum claritudo dierum solet imitari fulgorem, "voilà ce qu'il avait l'audace de faire dans une ville oủ la clarté des lumières nocturnes égale d'ordinaire l'éclat du jour” (trad. Coll. Univ. France, E. Galietier - J. Fontaine, 1968). Jean-Charles Balty m'a aimablement indiqué qu'une inscription, inédite, d’Apamée-sur-l'Oronte fait mention de l'éclairage public de la ville. Voir aussi M. MossakowskA, «Les huiles utilisées pour l'éclairage en Égypte (d'après les papyrus grecs)", Journal of Juristic Papyrology, 24, 1994, pp. 101-139.

(17) E. Stein, Histoire du Bas-Empire, 11, Amsterdam, 19682, pp. 192-215. Pour l'intérêt tout particulier de l'empereur Anastase pour les spectacles de l'hippodrome, cf. A. CAmEron, Porphyrius the Charioteer (op. cit.), p. 241 passim.

(18) Des exemples concrets, pour d'autres villes d'Orient, sont étudiés dans L'origine des richesses dépensées dans la ville antique (Colloque 1984), Aix-en-Provence, 1985. Pour l'Égypte, D. BonneAu, "Aperçu sur l'origine de la richesse des villes antiques en Égypte d'après la documentation papyrologique», pp. 233-245, n'évoque pas la question du financement public mais constate aussi que sur la question de l'origine de la richesse des villes, les sources papyrologiques restent inexploitées. Pour la notion de «ville», plus fluctuante en Égypte qu'ailleurs (cité grecque, polis, métropole de nome, gros bourg, village, etc.), cf. G. WAGNER, «L'épigraphie du village dans l'Égypte grecque et romainen, L'epigrafia del villaggio, Faenza, 1993, pp. 101-116. 
Au cours du $\mathrm{vI}^{\mathrm{e}} \mathrm{S}$., les villes engagent des sommes considérables pour les spectacles de l'hippodrome, ce qui traduit l'importance décuplée prise par les courses de chevaux dans la vie municipale dès le règne de Justinien.

Il peut paraître étrange de ne pas trouver mention de l'hippodrome dans CP Herm. 127 verso $=$ P. Vindob. Gr. 12565, qui est un long relevé de frais détaillés pour des travaux de restauration à effectuer sur des édifices d'Hermopolis - mais il est vrai que cette $\sigma \dot{v} v o \psi \iota \varsigma$ dressée par le bouleute Aurelius Appianus, ne concerne que le centre monumental de la ville $\left({ }^{19}\right)$.

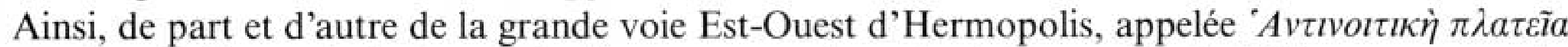
(Antinoopolis est située à peu de distance en aval sur la rive opposée du Nil), on a entrepris, vers le milieu du $\mathrm{m}^{\mathrm{e}}$ s., entre la Porte du Soleil et la Porte de la Lune, des travaux importants au temple de Thot-Hermès, au makellon, au temple d'Hadrien, au temple d'Antinoüs, au Sérapeum, au komasterion, au gymnase, à l'agora, etc. pour ne citer que les monuments religieux et publics les plus importants. Le coût total des travaux est impressionnant : 65 talents 4037 drachmes et 2 oboles. Rien pour l'hippodrome, mais l'examen des sources relatives aux couleurs des factions permettra peut-être de situer approximativement le monument dans la topographie urbaine. Au moment de ces grands travaux publics, en tout cas, il a existé (on a évoqué des courses de chevaux pour l'année 195). Mais on peut remonter un peu dans le temps, grâce à l'inscription de Seuthès. Cette épigramme trouvée en 1932/1933 aux abords immédiats du temple funéraire de Pétosiris dans le secteur sud de la grande nécropole de Tounah el-Gebel, est publiée $\left({ }^{20}\right)$ sous le titre «Le mort qui sentait bon», parce que Seuthès n'a pas voulu, selon le texte gravé par son cousin Philhermès, être embaumé à l'égyptienne mais a préféré des funérailles à la mode grecque. C'est l'épitaphe d'un garçon mort à 12 ans de phtisie. L'inscription peut être datée du second ou du troisième tiers du $\mathrm{II}^{\mathrm{e}} \mathrm{s}$., avec vraisemblance entre 130 et $180 / 190$, en s'appuyant sur le fait que l'agoranomie exercée par le grand-père et le père du défunt est encore présentée ici comme un honneur, ce qui pourrait témoigner en faveur de la datation proposée. Relevons cependant que dès la fin du $\mathrm{II}^{\mathrm{e}} \mathrm{s}$., en 192 précisément, dans

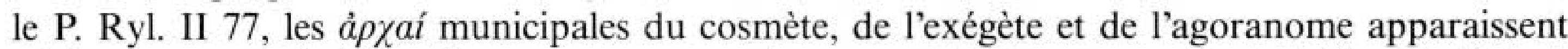
déjà comme un fardeau plus que comme un honneur. Cet écrasement total du liturge désigné devient courant au $\mathrm{ml}^{\mathrm{e}} \mathrm{s}$. Aux lignes 9 à 11 de l'inscription de Seuthès, qui concernent Épimaque, son père, il est fait mention des «innombrables victoires que remportent ses chevaux»; en

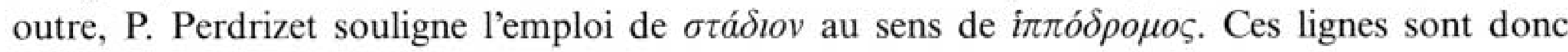
un témoignage supplémentaire de l'existence de l'hippodrome d'Hermopolis.

La possession d'une écurie de course suppose aussi d'importants revenus et peut donner des indices sur la fortune du personnage; cependant, la charge exercée par Épimaque, dans le courant $\mathrm{du} \mathrm{II}^{\mathrm{e}} \mathrm{s}$., est une liturgie, alors qu'au $\mathrm{VI}^{\mathrm{e}} \mathrm{s}$., date de notre papyrus, c'est la caisse municipale qui finance les courses de l'hippodrome.

D'autres mentions, plus tardives, de l'hippodrome, contiennent des indications à caractère topographique. Dans l'ordre chronologique, il convient de retenir les papyrus suivants (voir $C E, 58,1983$, pp. 226-228) :

(19) H. Schмiтz, «Die Bau-Urkunde in P. Vindob. Gr. 12565 im Lichte der Ergebnisse der deutschen HermopolisExpedition", Münchener Beiträge zur Papyrusforschung und antiken Rechtsgeschichte, 19, 1934, pp. 406-428. P. Vindob. Gr. $12565=S B, X, 10299$ est daté du 11 mai 263. Pour les fouilles à Hermopolis, voir : G. Roeder, Hermopolis 1929-1939, Hildesheim, 1959 ; A. J. SPENCER, Excavations at El-Ashmunein I: The Topography of the Site, Londres, 1984 ; D. M. BAILEy, Excavations at El-Ashmunein IV. Hermopolis Magna : Buildings of the Roman Period, Londres, 1991.

(20) P. Perdrizet, «Le mort qui sentait bon», Annuaire de l'Institut de philologie et d'histoire orientales (= Mél. J. Bidez), II, 1934, pp. 719-727. 
1. CPR VI 63, 1 (Hermopolis, déb. IV $\mathrm{v}$.), sans doute un ordre de paiement, concerne

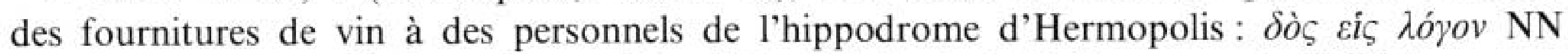

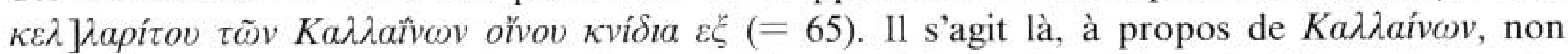
pas de faïences bleu turquoise, mais des partisans de la couleur bleue de l'hippodrome. Le papyrus concernerait donc un paiement à l'ordre d'un $\kappa \varepsilon \lambda \lambda$ apío de la faction des Bleus.

2. P. Strasb. 287, 1 (Hermopolis, $\mathrm{vl}^{\mathrm{e}} \mathrm{s}$.), est la reconnaissance de dettes d'une corporation $\tau \tilde{\eta} \varsigma$ ép du cirque, et non pas d'un cours d'eau comme l'avait pensé l'éditeur du papyrus.

3. BGU XII 2201 (Hermopolis, mars-avril 565?) est un prêt adressé à un nyctostratège,

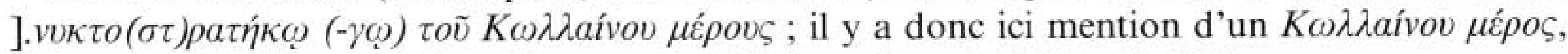

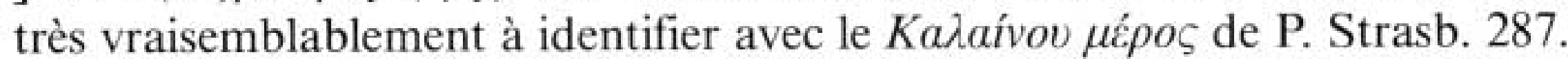

4. Il faut citer, parallèlement au texte $\mathrm{n}^{\circ} 3$ et pour tenter d'approcher d'une localisation approximative de l'hippodrome, un papyrus hermopolitain daté du 29 octobre 504 (P. Vindob.

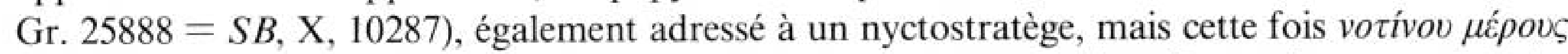

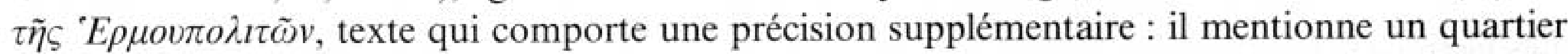
Sud d'Hermopolis; or, dans ce même quartier Sud, d'après P. Strasb. 287, étaient installées des corporations d'artisans. C'est toujours dans ce même quartier qu'est aussi située une église St-Théodore selon P. Sorb. II 69, 44 ( $\mathrm{f}^{\circ} 22$ ), 26, un codex fiscal hermopolite du début du

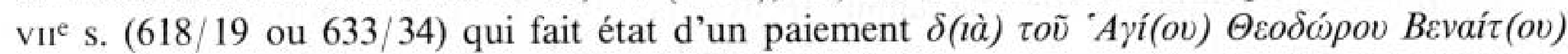

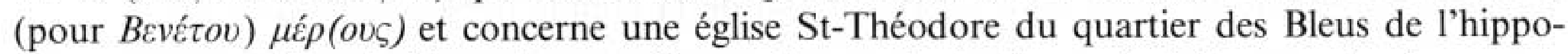
drome ; ce quartier, placé sous la surveillance d'un nyctostratège, qui abrite en outre des corporations d'artisans à propos desquels on évoque la part des Bleus du cirque, serait donc à localiser dans la partie méridionale de la ville.

Trois textes maintenant pour la couleur verte.

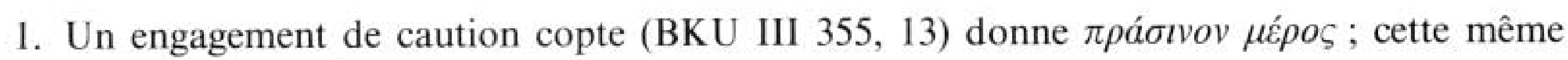
couleur verte est mentionnée par :

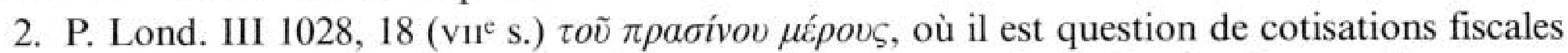
collectées, rue par rue, auprès d'habitants (artisans et membres du clergé) pour le compte de la "part verte». Puisque la partie inférieure de P. Lond. III 1028 est consacrée à la part verte, il est très probable, dans un compte aussi détaillé, que la partie supérieure, qui comporte aussi l'indication de rues et de sommes à y prélever, ait été consacrée à la part bleue.

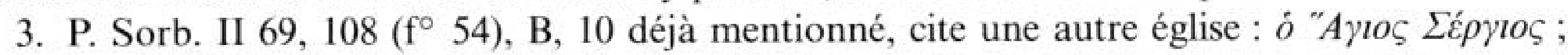
or, l'on sait, d'après P. Lond. III 1028, 23 qu'un certain Phoibammôn, économe de cet établissement, y a procédé à un paiement "pour la rue de St-Ménas», que le papyrus place dans ce même quartier : l'église St-Serge est donc située dans le quartier de la faction des Verts.

D'après ces différents textes, les quartiers des Bleus et des Verts - pour le premier, la chose parait certaine - sont à chercher dans la partie Sud d'Hermopolis et de toute façon au sud de la grande voie bordée de monuments religieux et publics, décrits par le P. Vindob. Gr. 12565 du milieu du ${ }^{\prime \prime} \mathrm{I}^{\mathrm{e}} \mathrm{s}$. On peut penser que c'est dans le secteur méridional de la ville que devait être situé l'hippodrome.

Le papyrus de Vienne P. Vindob. Gr. 15253 est un témoignage supplémentaire à verser au dossier des mentions de l'hippodrome d'Hermopolis mais aussi au difficile problème des finances municipales en Égypte byzantine. 